\title{
Total or Subtotal Thyroidectomy in the Management of Multinodular Goiter
}

\author{
Abdelaziz Ahmed Mohamed Gonna, MD. \\ Department of surgery, Shibin Elkoom Teaching Hospital, Egypt.
}

Background: For a long time, subtotal thyroidectomy was considered the standard surgical approach for multinodular goiter; however this concept started to change in favour of total thyroidectomy. Many issues have been involved in that argument before rendering one operation superior to the other. This work was conducted to compare between the two operations in terms of occurrence of postoperative morbidity and the functional outcome.

Patients \& methods: A total number of 106 patients with multinodular goiter were managed surgically in Gazan General Hospital. A group of 57 patients who underwnt subtotal thyroidectomy were compared with 49 patients who underwent total thyroidectomy. The obtained data included patient demographics, operation type, pathology, hospital stay and postoperative early and late complications such as recurrent laryngeal nerve palsy, parathyroid gland affection, wound infection, haematoma, reoperation for haemorrhage, and death.

Results: There was no significant difference in the sex, age, duration of goiter or hormonal status between the two groups ( $P=0.64, P=0.73, P=0.59$ and $P=0.73$, respectively). The compression symptoms and recent enlargement of the gland represented the two main indications for surgery in both groups. The mean operating time and the mean hospital stay was shorter in the subtotal group when compared with the total group but this was not statistically significant $(P>0.05)$. The difference in the incidence of transient and permanent nerve palsy between the two groups was not statistically significant $(P>0.05)$. The incidence of postoperative temporary hypocalcaemia was significantly higher in total thyroidectomy group $(P=0.02)$ while the incidence of permanent hypocalcaemia was not statistically significant between the two groups $(P>0.05)$.

Conculsion: The rate of permanent complications with total thyroidectomy is not greater than that of subtotal thyroidectomy so, it can be performed safely and should be recommended as the procedure of choice for patients requiring surgical treatment for multinodular goiter.

Key words: goiter, total thyroidectomy, subtotal thyroidectomy.

\section{Introduction:}

Goiter is the most common endocrine disorder requiring surgical management, especially in areas where iodine deficiency is prevalent. The World Health Organization (WHO) reported that $5 \%$ of the world's population suffer from goiter and that $75 \%$ of these people live in iodine deficient areas. ${ }^{1}$

Thyroidectomy remains the treatment of first choice for these patients with multinodular goiter especially with suspected malignancy and large-sized goitre causing symptomatic compression. ${ }^{2}$ The procedure of thyroidectomy was developed by Theodore Kocher and William Halsted. ${ }^{3}$ Since that time in the last century, it has developed from a life-threatening intervention to an efficient, wide-spread, and safe procedure nowdays. The mortality of thyroid surgery has been reduced to a very low percentage due to progress in the control of bleeding and experience in the management of tracheal problems. 4 Traditionally, benign multinodular goitre has been treated by subtotal thyroidectomy. However, in recent years, function and morphology-orientated 
surgery has been favoured. ${ }^{2}$ In 1997 Korun and his colleagues reported that $40 \%$ of the patients with multinodular goitre have nodules localized in the dorsal part of the gland, which are usually left during normal subtotal resection. ${ }^{5}$

Recently, total thyroidectomy has replaced bilateral subtotal thyroidectomy as the preferred option for the management of all patients with bilateral benign multinodular goitre, Graves' disease, all types of thyroid cancer ${ }^{6}$ and other less common conditions, such as thyroiditis and Amiodarone-induced thyrotoxicosis. ${ }^{3}$ Although total thyroidectomy provides predictable results, it is still debated by many authors because of the assumed higher complication rates. However, total thyroidectomy is now being performed widely throughout the world for benign euthyroid multinodular goitre with lowcomplication rate. ${ }^{7}$ To determine the ideal treatment, surgeons must weigh the risk of complications of a more aggressive surgical resection (total thyroidectomy) versus the risk of morbidity, mortality, recurrence rates and difficulty in following up patients who undergo less gland resection. ${ }^{8}$

The aim of the present study is to evaluate the safety of employing total thyroidectomy instead of subtotal thyroidectomy as the procedure of choice for patients requiring surgical treatment for multinodular goiter. Patients who was managed by subtotal thyroidectomy were compared with those managed by total thyroidectomy with reference to the occurrence of postoperative morbidity and the functional outcome.

\section{Patients and methods:}

A total number of 106 patients with multinodular goiter were subjected to surgical management in Gazan General Hospital and were included in the study during the period from April 2011 to April 2013 (24 months). 57 patients were assigned to have subtotal thyroidectomy and 49 patients were assigned to have total thyroidectomy.

The decision to choose one of the two procedures was based on the preoperative clinical and sonographic evaluation, intraoperative macroscopic findings and the pattern of nodular dissemination. Those patients who had no healthy tissue or had nodules localized in the dorsal part of the gland, which are usually left during normal subtotal resection or their fine needle aspiration cytology (FNAC) showed suspected malignancy were assigned to the total thyroidectomy group and the others were assigned to the subtotal thyroidectomy group.

For each patient, certain data were collected. It included patient demographics, surgical procedure, pathology, hospital stay and post-operative early and late complications including temporary hypoparathyroidism, permanent hypoparathyroidism, temporary recurrent laryngeal nerve paresis, permanent recurrent laryngeal nerve palsy, wound infection, haematoma, reoperation for haemorrhage, and death.

The preoperative assessement included thyroid ultrasound, determination of free $\mathrm{T}_{3}$, free $\mathrm{T}_{4}$, thyroid stimulating hormone (TSH) and (FNAC). A routine laryngoscopy was performed before the operation to all patients to assess the laryngeal nerve function but postoperative laryngoscopy was performed only to symptomatic patients as the surgeon's assessment of the patient's voice was a reliable method to suspect vocal cord palsy in the days after surgery. ${ }^{9}$ Serum calcium was measured routinely on all patients postoperatively and calcium supplementation was given only if symptoms of hypocalcaemia developed.

Total thyroidectomy entails removal of all the thyroid tissue using the capsular dissection technique while subtotal thyroidectomy is performed essentially in the same manner except that an intended 4-5 g remnant is left on both sides in the region of the ligament of Berry. ${ }^{10,11}$ Thyroidectomy was performed through a collar incision with the patient under general anaesthesia and endotracheal intubation. Elevation of superior subplatysmal flap till the thyroid notch was done while the inferior subplatysmal flap was not routinly elevated. The strap muscles were divided in the midline. The middle thyroid veins were ligated and divided early in the operation. The 
superior thyroid vessels were individually ligated and divided after identification and preservation of the superior laryngeal nerve when possible. Their division allowed the gland to ride up into the neck to a degree, greatly assisting delivery of the more inferior components of the goitre. The recurrent laryngeal nerves (RLN) were identified routinely in the tracheoesophageal groove during ligation of the inferior thyroid artery and every attempt was made to preserve it. The superior and inferior parathyroid glands were preserved with intact blood supply when possible. This capsular dissection was followed by either total or subtotal resection according to the case. The neck was routinely drained with bilateral rubber drains for $24 \mathrm{~h}$.

The patients were usually discharged 3-4 days postoperatively. During this period, measurement of serum calcium was performed at least once daily until stabilization during hospital stay. Replacement with calcium and/or vitamin D analogue was given when hypocalcaemic symptoms developed. Hypoparathyroidism was defined as sustained symptomatic hypocalcaemia with lower serum calcium levels.

Hypoparathyroidism was considered permanent, if the patient required calcium supplement to maintain normal serum calcium level for 6 months or longer. If the patient could be weaned from the calcium supplement within 6 months, the situation was accepted as transient hypoparathyroidism. ${ }^{1}$

Postoperative laryngoscopy was performed only to patients with voice changes. When vocal cord palsy was identified, the procedure was repeated every two months thereafter until recovery was achieved, with the dysfunction considered permanent if it still persisted after 6 months. ${ }^{12}$ After total thyroidectomy, $200 \mathrm{mg}$ of levothyroxine was commenced, while it was $100 \mathrm{mg}$ of levothyroxine for subtotal thyroidectomy and thereafter the dose of levothyroxine was adjusted according to the free $\mathrm{T}_{3}$, free $\mathrm{T}_{4}$ and TSH levels.

Patients who underwent subtotal thyroidectomy were compared with those who underwent total thyroidectomy with respect to demographics, operative details, postoperative complications and long-term outcome. Statistical analysis was performed with the SPSS/PC + (version 9).

\section{Results:}

Data gathered from patient charts included age at time of operation, gender, duration of the disease, preoperative hormonal assay, the indication for thyroidectomy, operative time, the type of surgery performed, the postoperative diagnosis as taken from the postoperative pathology report, and the postoperative copmlications as recurrent laryngeal nerve palsy, hypocalcaemia, wound infection, haematoma, reoperation for haemorrhage and death.

There were 57 and 49 patients in the subtotal and total thyroidectomy groups, respectively. The subtotal thyroidectomy group comprised of 11 men and 46 women with a mean age of 45 years \pm 10.6 while the total thyroidectomy group comprised of 9 men and 40 women with a mean age of 43 years \pm 11.2 . For patients undergoing subtotal thyroidectomy, the mean duration of goiter was 3.25 years \pm 2.68 while it is 4.12 years \pm 3.85 for the patients undergoing total thyroidectomy. According to thyroid functional status, the majority of patients were euthyroid in both groups $(87.7 \%$ in the subtotal group versus $91.8 \%$ in the total group). There was no significant difference in the sex, age, duration of goiter or hormonal status between the two groups $(\mathrm{P}=0.64, \mathrm{P}=0.73, \mathrm{P}=0.68$ and $\mathrm{P}=0.73$, respectively). The previous criteria are summarized in Table (1).

The compression symptoms and the recent enlargement of the gland represent the two main indications in both groups. In the subtotal group, the compressive symptoms accounts for $49.2 \%$ and the recent enlargement of the gland accounts for $35 \%$ while in the total group, the compressive symptoms accounts for $39.9 \%$ and the recent enlargement of the gland accounts for $35.6 \%$. The main compressive symptoms were neck discomfort, breathing and swallowing difficulties. The toxic manifestations and suspected cancer are less common indications. The indications 
for surgery are presented in Table (2).

The operating time was defined as the time passed from the first incision to the last skin suture placement. The mean operating time was shorter in the subtotal group when compared with the total group (123.1 \pm 24.4 min. vs $132.6 \pm 33.8 \mathrm{~min}$. respectively). The mean hospital stay for patients who underwent subtotal thyroidectomy (3.1 \pm 2.3 days) was also shorter than those who underwent total thyroidectomy (3.3 \pm 2.1 days). Although the operating time and hospital stay were shorter in the subtotal group, this difference was not statistically significant between the two groups $(\mathrm{P}>0.05)$. Figure (1) shows the difference in the operating time and mean hospital stay in both groups.

The preoperative FNAC did not prove malignancy in any case but it suspected it in 8 patients. These patients with suspicious FNAC were assigned to have total thyroidectomy. Postoperatively, thyroid cancer was incidentally found in seven patients of both groups. Six of them were in the total group $(12.2 \%)$ while the remaining one was in the subtotal group $(1.8 \%)$. Five patients among the six patients that has cancer in the total group were suspected by the FNAC (83.3\%). The histopathological diagnosis was papillary carcinoma in five patients $(71.4 \%)$ and follicular carcinoma in two patients $(28.6 \%)$. The only patient that had cancer in the subtotal group was reoperated on later to excise what was left in the previous operation.

Six $(10.5 \%)$ and five patients $(10.2 \%)$ in the subtotal and total groups, respectively, developed transient vocal cord palsy after the operations. All had unilateral affection except one patient that was in the total group. However, her airway was patent and tracheostomy was not required. In all patients, the vocal cord function recovered spontaneously over a mean duration of 3.1 months (range, 1-6 months) after the operations. There was no permanent recurrent laryngeal nerve palsy in all patients. The difference in the incidence of nerve palsy between the two groups was not statistically significant $(\mathrm{P}>0.05)$.

In contrast, the incidence of postoperative temporary hypocalcaemia was significantly higher for patients who underwent total thyroidectomy compared to those who underwent subtotal thyroidectomy $(\mathrm{P}=0.02)$. Seven out of $57(12.3 \%)$ compared with eleven out of 49 patients $(22.4 \%)$ in the subtotal and total group, respectively, developed temporary hypocalcaemia in the early postoperative period. However, the parathyroid function recovered completely over a mean duration of 16 days \pm 3.8 . Only one patient devolped permenant hypocalcaemia that was not recovered over a peroid of 6 months. She was in the total group. However, the incidence of permanent hypoparathyroidisim was not statistically significant between the two groups $(\mathrm{P}>0.05)$.

Reoperation for haemorrhage was required for two patients $(3.5 \%)$ who underwent subtotal thyroidectomy, and for one patient (2\%) who underwent total thyroidectomy. Wound healing problems occurred in one patient in each treatment group which were treated conservetively. There was no statistically significant difference between the two groups with respect to wound infection, haematoma and reoperation for haemorrhage. There was no mortality in both groups. Figure (2) summarizes the postoperative complications in both groups.

\section{Discussion:}

For a long time, subtotal thyroidectomy was the gold standard operation for patients with benign multinodular goiter. It is believed that by leaving a small remnant of functioning thyroid gland, euthyroidism can be achieved without the need for lifelong thyroxine replacement. In addition, limited dissection is performed in the tracheooesophageal grooves with minimal potential risk of damaging the recurrent laryngeal nerves as well as the parathyroid glands. Therefore, it is thought to be a safer operation compared to total thyroidectomy with respect to postoperative recurrent laryngeal nerve palsy and hypoparathyroidism. However, this concept is being challenged by the excellent results achieved by an increasing number of endocrine surgeons who are adopting total 


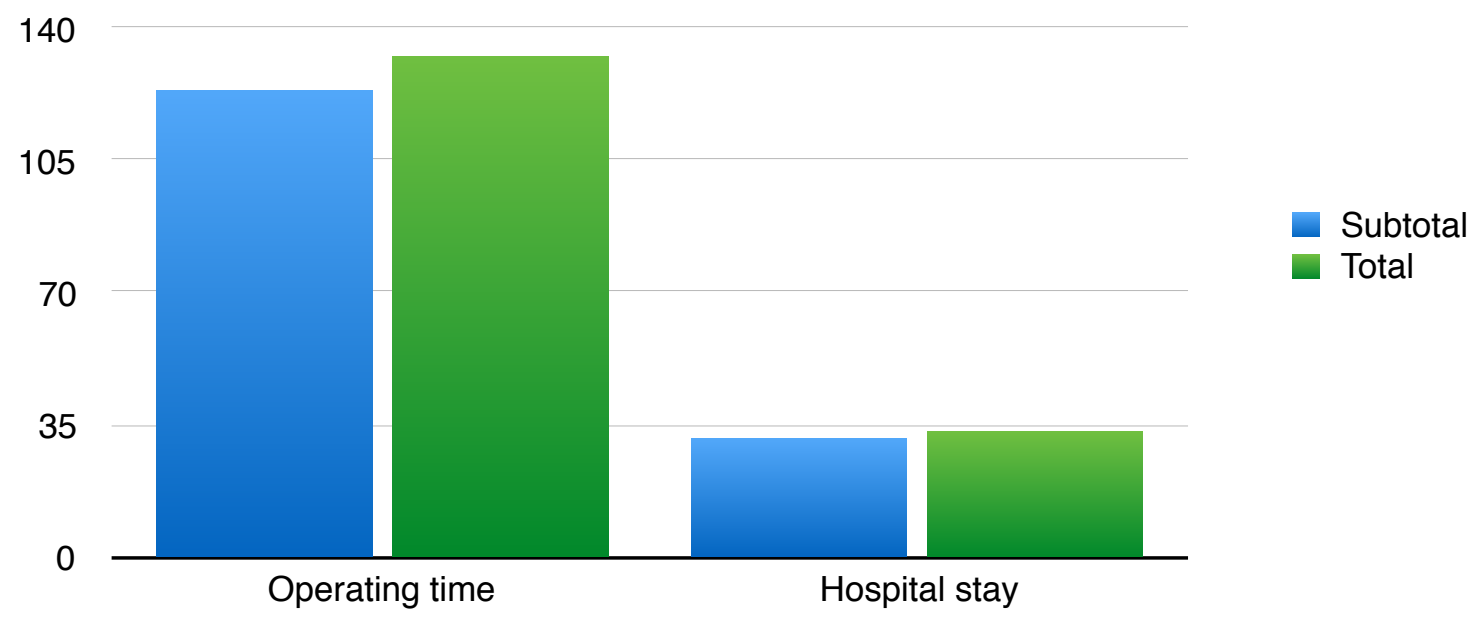

Figure (1): The difference in the operating time and mean hospital stay in both groups.

Subtotal Total

30

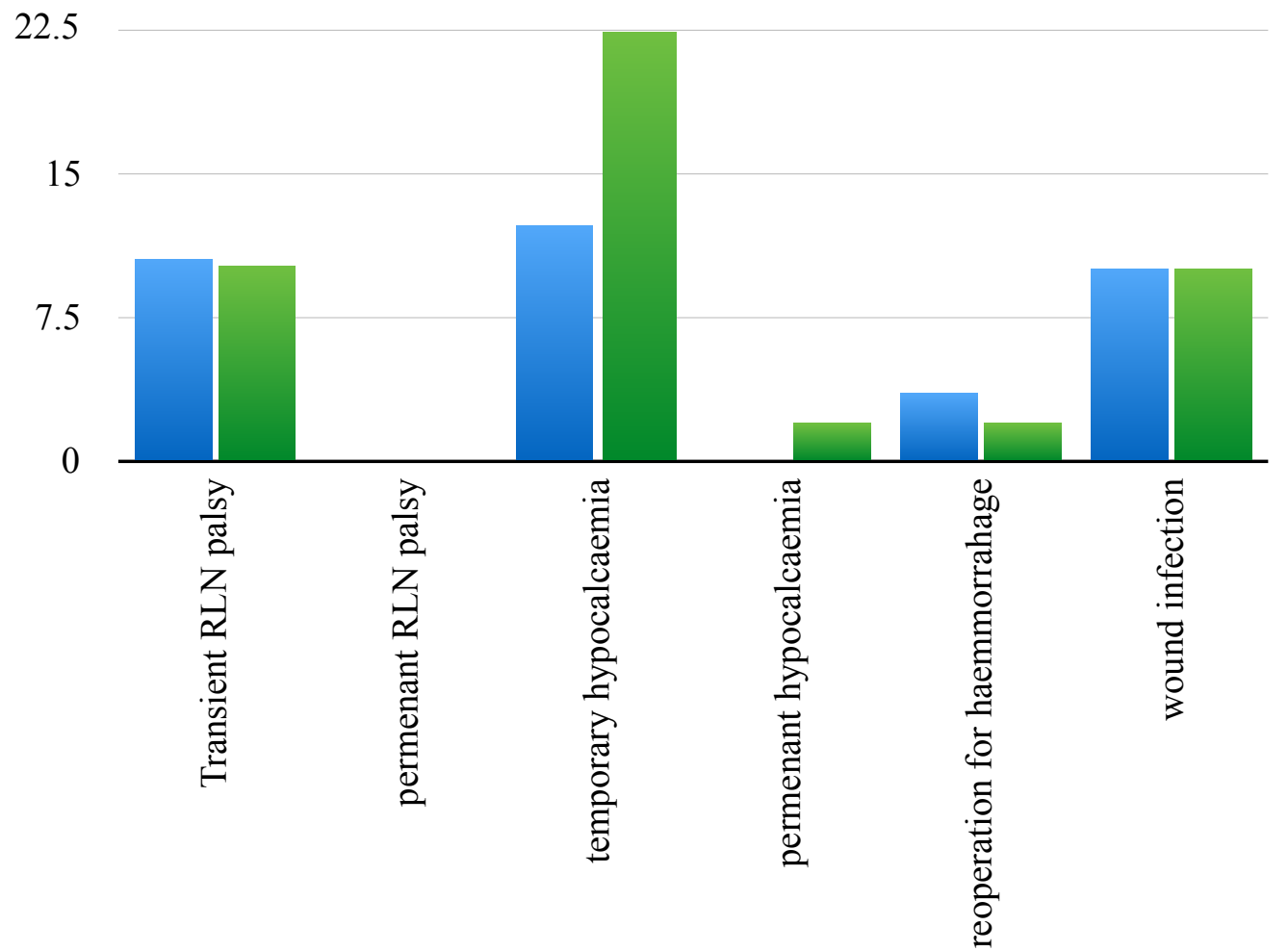

Figure (2): Postoperative complications in both groups.

Table (1): Clinical features of patients undergoing subtotal and total thyroidectomy.

\begin{tabular}{|l|l|l|}
\hline & \multicolumn{1}{|c|}{ Subtotal } & \multicolumn{1}{c|}{ Total } \\
\hline Number & 57 & 49 \\
\hline Sex ratio $(\mathrm{M}: \mathrm{F})$ & $1: 4.2$ & $1: 4.4$ \\
\hline Mean age (years) & 45 years \pm 10.6 & 43 years \pm 11.2 \\
\hline Mean duration of goiter (years) & 3.25 years \pm 2.68 & 4.12 years \pm 3.85 \\
\hline Euthyroid status & $87.7 \%$ & $91.8 \%$ \\
\hline
\end{tabular}


Table (2): Indications for surgery.

\begin{tabular}{|l|l|l|}
\hline & \multicolumn{1}{|c|}{ Subtotal } & \multicolumn{1}{c|}{ Total } \\
\hline Compression symptoms & $49.2 \%$ & $39.9 \%$ \\
\hline Recent enlargement of the gland & $35 \%$ & $35.6 \%$ \\
\hline Thyrotoxicosis & $15.8 \%$ & $8.2 \%$ \\
\hline Suspicious FNAC & $0 \%$ & $16.3 \%$ \\
\hline
\end{tabular}

thyroidectomy as the preferred option for the surgical management of benign multinodular goiter ${ }^{13}$ and now total thyroidectomy for benign thyroid disease is likely to become an accepted component of the endocrine surgeon's armamentarium. ${ }^{14}$

Another reason is that it is difficult to leave healthy tissue intact because the nodular dissemination reaches the dorsal capsule in many patients. It is accepted that when the entire gland is diseased in patients with multinodular goiter, a subtotal thyroidectomy, although reducing the bulk of the diseased tissue, is not an optimal treatment. ${ }^{15}$ It is also possible that the remaining tissue after performing subtotal thyroidectomy often extends into the retrotracheal and/or retroesophageal area, causing significant pressure symptoms. ${ }^{1}$

In 1995, Rojdmark and Jarhult studied the long-term recurrence rate after subtotal thyroidectomy and reported a $42 \%$ recurrence rate after 30 years follow up in patients who had a subtotal thyroidectomy because of multinodular goiter ${ }^{16}$ while in 1998, Pappalardo and his colleagues reported a $14.5 \%$ recurrence rate after subtotal thyroidectomy despite drug prophylaxis. ${ }^{17}$ Without drug suppressive therapy, the rate of recurrence rises to $43 \% .{ }^{18}$

Subtotal thyroidectomy was traditionally adopted as the procedure of choice at our departement in the belief that long-term euthyroidism can be achieved and that complication rates are less than that of total thyroidectomy. However total thyroidectomy is now being performed widely throughout the world for benign euthyroid multinodular goitre with low-complication rates 19 and started to be practiced widely in our department rendering the comparison between the two operations easy and clear.

Many issues have been involved in this comparison but it seems that the only real argument against total thyroidectomy is the potential for an increased risk of complications. However, there is good evidence to show that with increasing experience, total thyroidectomy can be performed with minimal complications. ${ }^{20}$

The two main complications are recurrent laryngeal nerve (RLN) injury and hypoparathyroidism. Both of them can occur in transient or permenant form. The frequency of recurrent laryngeal nerve (RLN) injury varies greatly from country to country, between different surgeons, with the extent of surgery and the nature of the initial disease. ${ }^{4}$ In the present study, the transient nerve injury ocurred in $10.5 \%$ and $10.2 \%$ in the subtotal and total group respectively which is not statistically significant. These results match with many previous studies like that of Colak T. and his colleagues who reported transient RLN injury in $9.3 \%$ of the patients with total thyroidectomy and $6.3 \%$ in the patients with subtotal thyroidectomy. ${ }^{1}$ Also the same results was obtained by another study conducted by Chun-Fan $\mathrm{Ku}$ and his colleagues in 2005. Although they reported higher incidence in the total group $(9.2 \%$ in the total versus $5.1 \%$ in the subtotal), yet both groups are still statistically insignificant. ${ }^{21}$ No cases with permanent (RLN) injury was reported in this study in both groups which renders no statistical difference between the two operations. The same conclusion was obtained by both Karanikolic A. 2007 and Barakate M. S. 2002 and in their studies.2,22

According to these results, we suppose that encountering the recurrent laryngeal nerve by use of the capsular technique of 
dissection and identifying the nerve close to the lateral thyrohyoid ligament is potentially safer than leaving it buried beneath residual posterior thyroid tissue while trying to achieve haemostasis of that residual tissue. Certainly, haemostasis is better achieved after total thyroidectomy when all vessels have been individually ligated outside the gland than when dealing with residual vascularized thyroid tissue.

Previous studies have reported that the incidence of temporary hypocalcaemia ranges from $1.6 \%$ to $22 \%$ after subtotal thyroidectomy and from $24 \%$ to $35 \%$ after total thyroidectomy $(17,23)$. In the present study, the incidence of temporary hypocalcaemia in total thyroidectomy (22.4\%) was significantly higher than that of the subtotal thyroidectomy $(12.3 \%)$. These results disagree with that of Colak T. and his colleagues 2004 and agree with that of Karanikolic A. and his colleagues 2007.1,2 This diversity in results may be due to different surgical techniques and different surgeons' experiences.

The incidence of permanent hypocalcaemia was $(0 \%)$ in the subtotal group and $(2.04 \%)$ in the total group. This result is comparable with the previous studies reporting incidence of permanent hypoparathyroidism ranging from $0.3 \%$ to $3 \%$ after total thyroidectomy. ${ }^{17,18,24}$ However it is still statistically insignificant between both groups.

We can conclude that with the exception of transient hypocalcaemia which is a temporary condition that usually resolves within an average period of two weeks, the risk of complications with total thyroidectomy is no greater than with subtotal thyroidectomy. So, we believe that nodular goitre should be managed by total thyroidectomy as the operation has a low rate of complications and minimizes the need for secondary surgery and its additional risks of morbidity.

\section{Reference:}

1- Colak T, Akca T, Kanik A, Yapici D, Aydin S: Total versus subtotal thyroidectomy for the management of benign multinodular goiter in an endemic region. ANZ Journal of Surgery 2004; 74 (11): 974-978.

2- Karanikolic A, Pesic M, Djordjevic N, Kocic
R, Radenkovic S, Stankovic D: Optimal surgical treatment for bilateral multinodular goitre. Surgical Practice 2007; 11(1): 12-16.

3- Serpell JW, Phan D: Safety of total thyroidectomy. ANZ Journal of Surgery 2007; 77(1-2): 15-19.

4- Schulte K, Röher H: Complications in the surgery of benign thyroid disease. European Surgery-Acta Chirurgica Austriaca 2001; 33(4): 164-172.

5- Korun N, Asci C, Yilmazlar T: Total thyroidectomy or lobectomy in benign nodular disease of the thyroid: Changing trends in surgery. Int Surg 1997; 82: 417-419.

6- Delbridge L: Total thyroidectomy: The evolution of surgical technique. ANZ Journal of Surgery 2003; 73(9): 761-768.

7- Mishra A, Agarwal A, Agarwal G, Mishra SK: Total thyroidectomy for benign thyroid disorders in an endemic region. World J Surg 2001; 25: 307-310.

8- Haddad A: Total thyroidectomy is the recommended treatment for all Papillary Thyroid Carcinoma (PTC). Acta Otorhinolaryngol Belg 1999; 53(3): 161-164.

9- Jarhult J, Lindestad PA, Noedenstrom J, Perbeck L: Routine examination of the vocal cords before and after thyroid and parathyroid surgery. Br J Surg 1991; 78: 1116- 1117.

10- Delbridge L, Reeve TS, Khadra M, Poole AG: Total thyroidectomy - the technique of capsular dissection. Aust ANZ J Surg 1992; 62: 96-99.

11- Bliss D, Gauger P, Delbridge L: The surgeons approach to the thyroid gland - anatomy and surgical technique. World J Surg 2000; 24 : 891-897.

12- Chiang FY, Wang LF, Huang YF, Lee KW, Kuo WR: Recurrent laryngeal nerve palsy after thyroidectomy with routine identification of the recurrent laryngeal nerve. Surgery 2005; 137(3): 342-347.

13- Razack MS, Lore JM, Lippes HA, Schaefer PD, Rassael H: Total thyroidectomy for Graves' disease. Head \& Neck 1997; 19: 378-783.

14- Wheeler MH: Total thyroidectomy for benign thyroid disease. Lancet 1998; 351: 1526-1527.

15- Reeve TS, Delbridge L, Cohen A, Crummer P: Total thyroidectomy: The preferred option for multinodular goiter. Ann Surg 1987; 206: 782-786.

16- Rojdmark J, Jarhult J: High long-term recurrence rate after subtotal thyroidectomy 
for nodular goiter. Eur J Surg 1995; 161: 725-727.

17- Pappalardo G, Guadalaxara A, Frattaroli FM, Illomei G, Falaschi P: Total compared with subtotal thyroidectomy in benign nodular disease: Personal series and review of published reports. Eur J Surg 1998; 164: 501-506.

18- Piraneo S, Vitri P, Galimberti A, Guzzetti S, Salvaggio A, Bastagli A: Recurrence of goiter after operation in euthyroid patients. Eur J Surg 1994; 160: 351-356.

19- Miccoli P, Vitti P, Rago T: Surgical treatment of Graves' disease: Subtotal or total thyroidectomy? Surgery 1996; 120: 1020-1024.

20- Gough IR, Wilkinson D: Total thyroidectomy for management of thyroid disease. World $J$ Surg 2000; 24: 962-965.

21- Chun FK, Chung YL, Wai FC, Annie WC, Kung SL: Total thyroidectomy replaces subtotal thyroidectomy as the preferred surgical treatment for Graves' disease. ANZ Journal of Surgery 2005; 75(7): 528-531.

22- Barakate MS: Total thyroidectomy is now the preferred option for the surgical management of Graves' disease. ANZ Journal of Surgery 2002; 72(5): 321-324.

23- Liu Q, Djuricin G, Prinz R: Total thyroidectomy for benign thyroid disease. Surgery 1998; 123: 2-7.

24- Chonkich GD, Petti GH Jr and Goral W: Total thyroidectomy in the treatment of thyroid disease. Laryngoscope 1987; 97: 397- 400. 